\title{
THE SPACES WHICH CONTAIN AN $S$-SPACE
}

\author{
W. F. PFEFFER
}

\begin{abstract}
Under the continuum hypothesis, we show that a $T_{1}$-space $X$ contains an $S$-space if and only if there is an uncountable locally countable set $E \subset X$ containing no Borel subset of $X$.
\end{abstract}

All spaces in this note are $T_{1}$. A space $X$ is called locally countable if each point has a countable neighborhood. A set $E \subset X$ is called locally countable if it is such as a subspace. An $S$-space is a hereditarily separable space which is not Lindelöf. We remark that contrary to the usual definition, in this note an $S$-space is not required to be regular. If $X$ is a space, we denote by $B(X)$ the family of all Borel subsets of $X$, i.e., the $\sigma$-algebra in $X$ generated by the topology of $X$. If $A$ is a set, we denote by $|A|$ its cardinality.

The Zermelo-Fraenkel set theory including the axiom of choice, the continuum hypothesis, and Martin's axiom will be abbreviated as ZFC, $\mathrm{CH}$, and MA, respectively.

1. Definition. A space $X$ is called ample if each uncountable locally countable set $E \subset X$ contains an uncountable subset $B \in B(X)$.

In this definition the word "ample" is used to indicate a certain richness of the Borel structure of $X$. The ample spaces are important in the topological measure theory; for each diffused, regular, Radon measure in an ample space is $\sigma$-finite (see $[$ [GP, 2.4]). Thus it appears useful to characterize the ample spaces in terms of much studied $S$-spaces.

The following lemma was first proved by R. J. Gardner (see [GP, 2.5]).

2. LEMMA. Let $E \subset X$ be a locally countable set containing no uncountable subset $B \in B(X)$. Then $E$ is hereditarily separable.

Proof. It suffices to show that $E$ is separable. By Zorn's lemma there is a maximal disjoint family $D$ of nonempty countable subsets of $E$ which are open in $E$. By the maximality of $D, \cup D$ is dense in $E$. For each $D \in D$, choose an $x_{D} \in D$, and let $B=\left\{x_{D}: D \in D\right\}$. Then $B \in B(X)$; for $B$ is discrete. By our assumption $B$ is countable, and hence so are $D$ and $\bigcup D$.

3. CoRollary. If $X$ is not ample, then it contains an $S$-space.

The following lemma is a special case of Theorem 2.6(i) from [J, p. 12].

4. LEMMA. Let $X$ be not Lindelöf. Then there is a locally countable $Y \subset X$ such that $Y$ is not Lindelof and $|Y|=\omega_{1}$.

Received by the editors November 4, 1981 and, in revised form, January 19,1982.

1980 Mathematics Subject Classification. Primary 54D20; Secondary 28A05.

Key words and phrases. Separable, Lindelöf, locally countable, Borel set, continuum hypothesis, Martin's axiom.

(c) 1982 American Mathematical Society 0002-9939/82/0000-0096/\$01.50 
Proof. Let $U$ be an open cover of $X$ which has no countable subcover. Then we can find a collection $\left\{U_{\alpha}: \alpha<\omega_{1}\right\} \subset \mathcal{U}$ where each $U_{\beta}-U_{\alpha<\beta} U_{\alpha} \neq \emptyset$. For every $\beta<\omega_{1}$, choose an $x_{\beta} \in U_{\beta}-\bigcup_{\alpha<\beta} U_{\alpha}$. The set $Y=\left\{x_{\beta}: \beta<\omega_{1}\right\}$ has the desired properties.

5. LEMMA. Let $Y$ be a hereditarily separable space with $|Y|=2^{\omega}$. Then there is an uncountable set $E \subset Y$ which contains no subset $B \in B(Y)$ with $|B|=2^{\omega}$.

ProOF. Let $\forall$ be the family of all sets $B \in B(Y)$ with $|B|=2^{\omega}$. Since $Y$ is hereditarily separable, it contains at most $|Y|^{\omega}=2^{\omega}$ closed subsets. Since $Y$ is a $T_{1 \text {-space, }|\not H|} \mid=2^{\omega}$. Now it is easy to construct a set $E$ with $|E|=2^{\omega}$ which contains no element of $\mathcal{H}$ (for the details see [K, $\S 40, I$, Theorem 2]).

6. THEOREM (CH). A space $X$ is ample if and only if it contains no $S$-space.

Proof. If $X$ contains an $S$-space, then by Lemma 4, it also contains a locally countable $S$-space $Y \subset X$ with $|Y|=2^{\omega}$. It follows from Lemma 5 that $Y$, and consequently $X$, is not ample. The converse is given by Corollary 3 .

The following example shows that Theorem 6 cannot be proved without $\mathrm{CH}$.

7. EXAMPLE. By an unpublished result of Szentmiklóssy there is a model $M$ of $\mathrm{ZFC}+\mathrm{MA}+\neg \mathrm{CH}$ in which a regular $S$-space exists. It follows from Lemma 4 that in $M$ there is a regular $S$-space $Y$ with $|Y|=\omega_{1}$. By [MS] (the lemma following Theorem 2 in \$2), in $M$ there exists a subspace $X$ of real numbers such that each subset of $X$ is Borel, and $|X|=\omega_{1}$. Choose a bijection $f: Y \rightarrow X$, and let $\tau$ be the weakest topology in $Y$ which refines the given topology of $Y$, and for which $f$ is continuous. Then each subset of $(Y, \tau)$ is Borel, and hence $(Y, \tau)$ is ample. However, since $X$ is second countable it is easy to see that $(Y, \tau)$ is still a regular $S$-space.

The author is obliged to the referee for valuable comments and suggestions.

\section{REFERENCES}

[GP] R. J. Gardner and W. F. Pfeffer, Are diffused, regular, Radon measures $\sigma$-finite, J. London Math. Soc. 20 (1979), 485-494.

[J] I. Juhass, Cardinal functions in topology, Math. Centre Tracts 34, Math. Centrum, Amsterdam, 1971.

[K] K. Kuratowski, Topology, Academic Press, New York, 1966.

[MS] D. A. Martin and R. M. Solovay, Internal Cohen extensions, Ann. Math. Logic 2 (1970), 143-178.

Department of Mathematics, University of CAlifornia, DaVis, California 95616

Current address: Departmen ' Mathematics, University of Petroleum and Minerals, Dhahran, Saudi Arabia 